\title{
Liderazgo educativo docente. Un modelo para su estudio, discusión y análisis*
}

Luis Enrique Riascos-Hinestroza

https://orcid.org/0000-0002-8703-1867

Universidad Americana de Europa

(Unade), México

luisenrique.riascos@unade.edu.mx

\section{Irene Becerril-Arostegui}

https://orcid.org/oooo-0001-9359-1310

Universidad Americana de Europa

(Unade), México

irene.becerril@aulagrupo.es

\section{Resumen}

El liderazgo ha sido estudiado a través de los tiempos por muchos autores, en diferentes escenarios y con distintos enfoques, estudios que permiten concluir que es efectivo en cuanto promueve la participación de las personas, genera confianza en los participantes de los equipos, incrementa la productividad y mejora las relaciones interpersonales. Uno de los escenarios de estudio ha sido el sector educativo, enfocado principalmente en el análisis de los efectos del liderazgo del directivo docente. Precisamente la finalidad de esta investigación es desarrollar un Modelo de Estudio de Liderazgo Educativo Docente que permita el análisis del liderazgo del docente de aula y su efecto en variables escolares. La investigación consta, primero, de una revisión bibliográfica exhaustiva; segundo, del estudio y análisis de propuestas para el modelo; tercero, del estudio exploratorio de viabilidad del modelo; cuarto, de la validación del modelo por parte de expertos; finalmente, el instrumento es sometido a pruebas, en las que participan docentes y estudiantes. Como resultado de la investigación se obtiene el Modelo y su incidencia, conformado por 15 variables, clasificadas de primer nivel y segundo nivel y de efecto o resultado, cada una de ellas definida conceptual y operacionalmente de acuerdo con el contexto educativo; por último, al Modelo se le asocia un instrumento conformado por 82 preguntas que permiten medir cada una de las 15 variables.

\section{Palabras claves (Fuente: Tesauro de la Unesco)}

Docencia; innovación educacional; liderazgo; modelo educativo; transformación social.

* $\quad$ Este artículo es producto de la tesis doctoral en Investigación y Docencia “Liderazgo educativo en instituciones oficiales de la zona rural de Tuluá, Valle del Cauca-Colombia”, Universidad Americana de Europa (Unade).

Recepción: 21/04/2021 | Envío a pares: 15/05/2021 | Aceptación por pares: 7/06/2021 | Aprobación: 27/07/2021 


\title{
Teacher Educational Leadership. A Model for Study, Discussion, and Analysis*
}

\begin{abstract}
Leadership has been studied by many authors through ages, in different settings and from various approaches. These studies have concluded that leadership is effective because it promotes participation, makes teams confident, increases productivity, and improves interpersonal relationships. Education has been one of the studied settings, focusing mainly on the managing teacher's leadership effect. This research aims to develop a Teacher Educational Leadership Study Model to analyze classroom teachers' leadership and its effect on school variables. Ths research passes through the following stages: 1) exhaustive literature review; 2) study and analysis of proposals for the model; 3) an exploratory study of the model's viability; 4) model validation by experts; and 5) instrument testing by teachers and students. As a result, we obtained the model and its impact, composed of 15 variables classified as first- and second-level and effect/outcome variables (teacher's leadership styles). Then, each variable is defined conceptually and operationally according to the educational context. Finally, an instrument formed by 82 items that measure the 15 variables is associated with the model.
\end{abstract}

\section{Keywords (Source: Unesco thesaurus)}

Teaching; educational innovation; leadership; educational model; social transformation.

\footnotetext{
* This article derives from the Research and Teaching dissertation "Liderazgo educativo en instituciones oficiales de la zona rural de Tuluá, Valle del Cauca-Colombia," Universidad Americana de Europa (Unade).
} 


\section{Liderança educacional docente. Um modelo para seu estudo, discussão e análise*}

\section{Resumo}

A liderança tem sido estudada ao longo do tempo por muitos autores, em diferentes cenários e abordagens. Esses estudos permitem concluir que ela é eficaz na medida em que promove a participação das pessoas, gera confiança nos participantes da equipe, aumenta a produtividade e melhora o relacionamento interpessoal. Um dos cenários de estudo tem sido o educacional, voltado principalmente para a análise dos efeitos da liderança da direção docente. Nesse contexto, o objetivo desta pesquisa é desenvolver um modelo de estudo de liderança educacional de professores que permita a análise da liderança do docente na sala de aula e seu efeito nas variáveis escolares. A pesquisa desenvolve-se nas seguintes etapas: revisão bibliográfica exaustiva; estudo e análise de propostas para o modelo; estudo exploratório de viabilidade do modelo; validação do modelo por especialistas; finalmente, o instrumento associado ao modelo é submetido a testes dos quais participam professores e alunos. Como resultado da pesquisa, há um modelo para o estudo da liderança educacional de professores e sua incidência, composto de 15 variáveis, classificadas como variáveis de primeiro nivel e segundo nível, e de efeito ou resultado; cada uma dessas variáveis é definida conceitual e operacionalmente de acordo com o contexto educacional. Por último, o modelo está associado a um instrumento composto de 82 questões, que permitem medir cada uma das 15 variáveis.

\section{Palavras-chave (Fonte: tesauro da Unesco)}

Ensino; inovação educacional; liderança; modelo educacional; transformação social.

* $\quad$ Este artigo é resultado da tese de doutorado em Pesquisa e Docência, intitulada "Liderança educacional em instituições oficiais da área rural de Tuluá, Valle del Cauca, Colômbia”, Universidad Americana de Europa (Unade). 
El liderazgo es un fenómeno social que ha sido estudiado en diferentes disciplinas, como la sicología, la historia, la sociología, la política y el desarrollo organizacional (Kroec et al., en Castro et al., 2007; Jiménez, 2008), de modo que su definición y concepción es diversa. El concepto de liderazgo y la concepción de líder se han modificado asimismo a través de los tiempos (Gómez, 2002). Este fenómeno ha sido entendido como rasgo personal, conducta, forma de interacción social o influencia o ha sido relacionado con el carácter del individuo líder (Gómez, 2002; Daft, 2006; Castro et al., 2007; Munroe, 2014). En conclusión, su conceptualización es diversa, depende del contexto en el que se estudie, se ha modificado con el tiempo y no existe un concepto único que lo abarque (Bass, 1990; Castro et al., 2007; Zalles, 2011; Navarro, 2016).

Tabla 1. Enfoques y tipos de liderazgo

\begin{tabular}{|c|c|}
\hline Enfoque o criterio & Teoria y tipos de liderazgo \\
\hline $\begin{array}{l}\text { Características } \\
\text { personales }\end{array}$ & $\begin{array}{c}\text { Teoría de los rasgos: liderazgo } \\
\text { carismático y liderazgo } \\
\text { motivacional. }\end{array}$ \\
\hline $\begin{array}{l}\text { Comportamiento } \\
\text { del líder }\end{array}$ & $\begin{array}{c}\text { Teorías conductuales: liderazgo } \\
\text { transaccional y liderazgo } \\
\text { transformacional. }\end{array}$ \\
\hline Rol social & $\begin{array}{c}\text { Teoría racional: liderazgo } \\
\text { burocrático. }\end{array}$ \\
\hline $\begin{array}{l}\text { Condiciones del } \\
\text { contexto }\end{array}$ & $\begin{array}{l}\text { Teoría situacional o de } \\
\text { contingencia. }\end{array}$ \\
\hline $\begin{array}{l}\text { Como fenómeno } \\
\text { conceptual }\end{array}$ & $\begin{array}{c}\text { Teoría de la atribución del } \\
\text { liderazgo. }\end{array}$ \\
\hline
\end{tabular}

Fuente: elaboración propia, basado en Gómez (2002).

Se pueden encontrar diferentes teorías, enfoques o perspectivas de estudio y distintas tipologías de liderazgo, sin que las descritas en la Tabla 1 constituyan los únicos tipos que los estudiosos han abordado, pues también se hace referencia al liderazgo distribuido, al burocrático y al democrático o participativo. Además, la aparición de nuevas complejidades hace que dichas teorías no sean suficientes para explicar la dinámica del liderazgo (Hurtado,
2019). Por último, debemos señalar que el liderazgo se ha analizado en diferentes escenarios: religioso, político, militar, económico y educativo, entre otros.

Respecto al liderazgo educativo, es preciso resaltar varios aspectos. Primero, este ha sido estudiado más respecto del rol del directivo docente que del docente de aula. Segundo, para el caso colombiano, se cuenta con poco desarrollo en la temática y su estado es incipiente (Barbosa et al., 2018; Sandoval et al., 2020). Tercero, se evidencia un rezago o desinterés del país en cuanto a producción científica relacionada con el tema, en comparación con otras regiones del mundo (Flessa et al., 2017), así como una baja producción en literatura internacional (Castillo y Hallinger, 2018).

No obstante, poner la lupa en el liderazgo escolar puede movilizar a las escuelas hacia el mejoramiento continuo de todos los procesos, ya que existen evidencias del vínculo entre liderazgo y buenos resultados escolares (Leithwood y Jantzi, 2000; Leithwood y Mascall, 2008; Horn y Marfán, 2010; Robinson, Lloyd y Rowe, 2014; Hernández, Murillo e Hidalgo, 2017). Además, esta es una de las competencias más importantes y necesarias en la gestión (Bedoya, Murillo y González, 2020). Pero los estudios no solo han estado orientados a señalar la existencia de alguna relación entre el liderazgo y los logros académicos de los educandos, sino también su vínculo con el desempeño de los docentes, el compromiso de los estudiantes y la motivación, entre otros asuntos. Hernández et al. (2017) asocian el liderazgo escolar con el hecho de facilitar el aprendizaje integral en todos los niveles de la escuela.

Entre los estudios teóricos cuya finalidad es dar cuenta del estado de la investigación en la temática, Horn y Marfán (2010) indagan por estudios empíricos que relacionen el liderazgo con el desempeño escolar en Chile, en 14 publicaciones del año 2000 en adelante. Los hallazgos de Robinson et al. (2014) buscan determinar el impacto de los diferentes tipos de liderazgo en los resultados académicos y no 
académicos de los estudiantes. Con este criterio, entre 1978 y 2006 se encontraron 27 estudios en varios países, la mayoría en Estados Unidos, pero ninguno en Latinoamérica. Dichos estudios, según los autores, muestran una relación directa del liderazgo con procesos de innovación o mejora educacional. De estos estudios, 23 estaban centrados únicamente en el liderazgo del director escolar o en quien desempeñase el rol directivo y 4 en el director y los docentes, pero ninguno centrado solo en el docente de aula.

Por su parte, los trabajos de Flessa et al. (2017) describen estudios sobre liderazgo escolar en Latinoamérica llevados a cabo entre los años 2000 y 2016 e incluyen documentos de políticas públicas relacionadas con el tema. Se encontraron 359 documentos, la mayoría en publicaciones de Chile (más de 100), Brasil y México. Para el caso colombiano, se encontraron 10 documentos, y se resalta la labor de la Fundación Empresarios por la Educación (FEXE) en apoyo del liderazgo escolar. A diferencia de Flessa et al., los estudios de Castillo y Hallinger (2018) tuvieron como finalidad revisar el estado del arte en la región producido en inglés y encontraron 48 estudios entre 1991 y 2017, donde 19 correspondían a Chile y 2 a Colombia.

Metodológicamente, el estudio se realiza en varias etapas. La primera consta de la revisión de literatura. La segunda atiende al diseño de propuestas para el modelo de estudio y análisis del liderazgo docente. La tercera presenta un estudio exploratorio poblacional. La cuarta valida el instrumento por expertos. Finalmente, el instrumento se somete a pruebas relacionadas con la comprensión de cada pregunta por parte de los potenciales encuestados y los tiempos requeridos para responderlo. El instrumento está basado en el modelo Full Range Leadership (FRL) de Avolio, Bass y Jung (1999).

\section{Problema y objetivo de investigación}

Este artículo se presenta como uno de los hallazgos de tesis doctoral que, como problema de investigación, asume una postura crítica de la calidad educativa cuando solo se analiza a través de resultados académicos medidos por pruebas estandarizadas. La calidad educativa se relaciona con el diario vivir escolar y por eso ellas no podrán generar información suficiente de los procesos de calidad en la escuela (Avendaño, Paz y Parada, 2016). La calidad educativa está asociada a muchos factores, entre los que se puede destacar el desempeño docente (Escribano, 2018), pero hay otros elementos o indicadores de calidad educativa, como la formación y liderazgo docente.

Este elemento de calidad (el liderazgo docente) puede indicidir en otros factores, también indicadores de calidad escolar, como: asistencia a clases, baja deserción escolar, participación de las familias, satisfacción escolar, efectividad de los procesos escolares, esfuerzo de los estudiantes, entre otros. En este sentido, surge la necesidad de estudiar el liderazgo educativo docente y su incidencia y con ello el desarrollo de un modelo para su discusión y análisis. Al problema planteado también se le suman los pocos estudios relacionados con liderazgo educativo centrado en el docente que se dan en el país.

Por lo anterior, se aborda como pregunta de investigación qué modelo permite estudiar mejor el liderazgo docente y su incidencia en variables escolares. Dicho de otra manera, la investigación tiene como obejetivo el desarrollo de un modelo que incluya diferentes tipologías o estilos de liderazgo, conductas o variables, que permitan el estudio y análisis del liderazgo docente en la educación regular. Finalmente, se tiene la necesidad de analizar la confiabilidad y correlacionalidad del modelo, como parte de las pretensiones de esta investigacipon empírica.

\section{Marco teórico-conceptual}

El sustento teórico del presente estudio está en el modelo Full Range Leadership (FRL), el cual incluye diferentes tipologías de liderazgo. El modelo tiene especial relevancia, pues el ejercicio de lideraz- 
go puede ser resultado de la amalgama de diversas conductas que originan diferentes liderazgos (Mendoza y Ortiz, 2006). El FRL incluye los componentes del liderazgo educativo transformacional (LET), liderazgo educativo transaccional (LES) y liderazgo educativo correctivo evitador (LECE). Del mismo modo, contiene los componentes conductuales relacionados con un bajo liderazgo o ausencia del mismo. Inicialmente, el modelo fue desarrollado por Bass en Leadership and performance beyond expectations (1985), donde presenta los hallazgos de las investigaciones relacionadas con el tema.

El FRL defiende la tesis de que el líder puede presentar varios estilos de liderazgo, desde el más efectivo hasta el altamente evitativo (Bass, 1985; Bass y Avolio, 1990, en Lee y Jennsen, 2014). Los líderes efectivos poseen características y rasgos diferenciadores, como lo muestran los resultados de las investigaciones de Bass (1985), donde se hace una descripción clara del liderazgo transformador y de las variables asociadas a este y se describe una herramienta o cuestionario para identificar a dichos líderes. Para Bass, el líder transformacional anima a las personas a hacer más de lo que de ellas se espera. Asimismo, estos líderes amplían y combinan los intereses de las personas a su cargo y simultáneamente generan conocimiento y aceptación respecto de los objetivos y la misión del equipo (1985, en García, 2015, p. 68).

El FRL conforma un todo y brinda multiplicidad de opciones de análisis y resultados en el ejercicio del liderazgo. Primero, porque permite estudiar la existencia de conductas que pueden ser clasificadas como indicadoras de niveles de liderazgo altos, medianos y bajos e incluso de no liderazgo o liderazgo incipiente, y segundo, por los resultados del liderazgo en lo que se refiere a: satisfacción de las necesidades de los individuos y del grupo, el esfuerzo extra requerido para el logro de los objetivos y la eficacia de la organización o el equipo (Mendoza y Ortiz, 2006, p. 120). Lo anterior hace del modelo una opción adecuada para estudios de liderazgo en diferentes contextos, pues permite contemplar no solo diversas tipologías de liderazgo, sino también sus efectos.

Es preciso resaltar que el modelo FRL incluye los conceptos y variables del LET y LES. De acuerdo con Avolio, Bass y Jung (1999, p. 441), las variables del modelo inicial son siete: carisma (C), inspiración (I), estimulación intelectual (EI), consideración individualizada $(\mathrm{Cl})$, recompensa contingente $(\mathrm{RC})$, gestión por excepción (GE) y liderazgo laissez-faire (LF). No obstante, en investigaciones posteriores, Bass concluyó que el liderazgo carismático e inspirador, por definición, eran lo mismo; por lo tanto, el modelo se redujo a seis variables (1988, en Avolio et al., 1999).

No obstante, estudios posteriores a los de Bass $(1985 ; 1988)$ encontraron fallas en el modelo de seis variables. Dichas fallas principalmente obedecían a la estructura de preguntas que medían las diversas variables. De acuerdo con Vega y Zabala, el cuestionario incorporaba en la misma escala de estudio preguntas que medían el impacto del comportamiento del líder en el seguidor, así como preguntas que solo medían comportamientos del líder (2004, p. 18). Por ello, los hallazgos de las investigaciones sugirieron separar la GE en activa y pasiva. Por su lado, Vega y Zabala señalan también que las variables de LF tenían mayor significancia correlacional con las preguntas de la escala GE pasiva (2004, p. 18).

\section{Compontentes del modelo FRL}

En el modelo FRL el liderazgo está conformado por comportamientos y atributos. Para Sosik y Jung (2018), el comportamiento se refiere a algo que la persona hace o dice y puede ser observado, descrito y registrado, mientras que el atributo es asignado por otros de acuerdo con lo que se percibe de la persona. Los comportamientos son acciones, los atributos son características o rasgos personales.

A continuación se describen las variables o factores del modelo FRL, el cual inspira la propuesta de análisis de un Modelo de Estudio del Liderazgo Educativo Docente (MELED). El FRL sugiere la existencia de 
múltiples estilos de liderazgo en una institución u organización (Lee y Jensen, 2014) y considera que los contextos educativos, por su dinámica, requieren diferentes estilos de liderazgo del docente.

Liderazgo educativo transformacional (LET). Implica conductas de alto nivel que permiten la transformación de procesos, estrategias y el quehacer de un equipo u organización, en procura de generar cambios profundos y positivos para todos. Quien lidera se distingue por poseer un alto carisma, al tiempo que las personas se identifican con el líder, a quien desean seguir y emular (Vega y Zabala, 2004). Asimismo, el líder es intelectualmente estimulante, enseña e inspira a los integrantes del equipo a través del desafio y la persuación, y les proporciona apoyo, guía y entrenamiento (p. 22). Como puede leerse, el LET se da en la interacción armónica entre personas y además ejerce influencia directa en la cultura organizacional (Santos, 2016). Lo anterior hace del LET una herramienta importante en el ámbito organizacional e institucional, pues los resultados en toda institución dependen de las personas y sus interacciones (Schmelkes, 2011).

Los estudios han mostrado una mayor efectividad del liderazgo transformacional (LET), comparado con el transaccional (LES). Por ejemplo, Bass (1990), McCleskey (2014) y Sosik y Jung (2018) muestran que los líderes transformacionales tienen mayores posibilidades de ser vistos como líderes eficientes y efectivos, al igual que permiten e inspiran que las personas hagan más de lo que deben hacer, realicen esfuerzos extras, trasciendan sus propios interesés y generen cambios significativos. Estos estudios también indican que el LES es apropiado en condiciones estables, sin mayores turbulencias, en tanto que el LET es propicio en condiciones que impliquen grandes cambios y transformaciones, y en situaciones adversas.

Liderazgo educativo transaccional (LES). Está basado en la recompensa, en los acuerdos, en la transacción. El líder ofrece beneficios o dádivas a cambio del cumplimiento del deber por parte de las personas a cargo, quienes acuerdan con este roles y responsabilidades interconectados para alcanzar metas establecidas, así como qué tareas o actividades deben realizar para obtener la recompensa o evitar la sanción acordada (Vega y Zabala, 2004, p. 106). De acuerdo con Contreras y Barbosa (2013) y Arévalo et al. (2015), el LES se caracteriza por: negociar la innovación, influenciar verticalmente, centralizar el poder, evitar la incertidumbre y centrarse en las tareas. Es un liderazgo positivo.

Estos intercambios, de acuerdo con McCleskey (2014), permiten a los líderes el logro de objetivos y tareas. De igual manera, la transacción lleva a motivar a las personas, lograr las metas establecidas y evitar riesgos. También se ha evidenciado que existe una relación directa entre el LES y el compartir conocimiento (Durán y Castañeda, 2015). En últimas, la estrategia del LES consiste en recompensar a las personas por el esfuerzo y buen desempeño o sancionar los malos resultados (Jensen et al., 2019). Finalmente, es de aclarar que el líder transformacional puede tener conductas del LES.

Liderazgo educativo correctivo evitador (LECE). Se caracteriza por que el líder monitorea las fallas en el desempeño, interviene solo cuando los problemas son graves y las acciones tomadas son de tipo correctivo (Vega y Zabala, 2004, p. 131).

Carisma e influencia idealizada atribuida (CIA) y conductual (CIC). Se presenta cuando el líder es un modelo a seguir y anima al equipo a lograr una visión y unos objetivos comunes, planteando las metas deseadas de manera clara y sencilla (Muenjohn, 2008). Vega y Zabala (2004) señalan que el líder hace sacrificios por el bienestar del quipo, se conserva sereno ante situaciones de adversas, busca solución a los problemas y, entre su propio interesés y el bienestar del equipo, prioriza el último. Su capacidad para generar confianza hace que las personas a cargo sobrepasen obstáculos y desborden talentos extraordinarios. Finalmente, como con- 
ducta, la influencia idealizada permite que el líder inculque valor, obtenga el respeto de los seguidores y demuestre poder y confianza. Los líderes carismáticos presentan un alto comportamiento moral. Sosik y Jung (2018) refieren, además, que estos poseen un férreo carácter y una sólida ética laboral, así que remiten permanentemente a la importancia de los valores, las creencias y los propósitos, así como a la misión colectiva.

Estimulación intelectual (EI). La El permite que las personas desarrollen la creatividad, la capacidad de innovación y el ingenio. Para Muenjohn (2008), los líderes tienen la capacidad de desafiar y llevar a los seguidores al máximo rendimiento. Presentan alto nivel de expectativa y proporcionan a los miembros del equipo tareas significativas y desafiantes. La El está estrechamente relacionada con la influencia idealizada y constituye un comportamiento que implica desarrrollar y articular una visión (Sosik y Jung, 2018).

Motivación inspiracional (MI). Está relacionada con la capacidad de los líderes de motivar y ser fuente de inspiración para cada uno de los miembros del equipo. De acuerdo con Vega y Zabala (2004), esta capacidad permite que el líder pueda generar una visión de equipo, enfatice en metas deseables para las personas y establezca de manera clara la forma o las estrategias para lograr dichas metas. Lo anterior hace que las personas se sientan incluidas e importantes. Asimismo, de acuerdo con Yukl y Van Fleet (1982, en Bass, 1985; 1990), la capacidad inspiradora de los líderes implica inculcar orgullo en los individuos, usar un lenguaje positivo y animador, ser ejemplo con sus propias aptitudes, inspirar entusiasmo y resaltar el buen desempeño de las personas. Los líderes que inspiran consiguen resultados óptimos, al lograr en las personas a cargo mayor compromiso y sentido de pertenencia. En Bass (1990) se encontraron cualidades de líderes inspiradores, como confianza, autoestima, autoderminación, sentido de pertenencia y compromiso por la misión.

Consideración individualizada (CI). Los líderes se concentran en enseñar y preparar a las perso- nas a cargo o seguidores (Muenjohn, 2008). El líder atiende las individualidades y necesidades de cada una de las personas a cargo.

Recompenza contingente (RC). Se refiere al intercambio material o no de dádivas, que obece al cumplimiento de tareas por parte de las personas a cargo. El buen desempeño se ve reflejado en premios y el malo en sanciones. Según Sosik y Jung (2018), la RC constituye un comportamiento de líder activo y efectivo y representa una relación de intercambio entre las partes.

Dirección por excepción activa (DEA). Esta conducta corresponde a líderes que se dedican a inspeccionar la realización de tareas y a detectar y corregir errores (Vega y Zabala, 2004; Muenjohn, 2008).

Dirección por excepción pasiva (DEP). El líder no hace seguimiento ni interviene en los procesos o tareas, a menos que no se consigan los objetivos. Espera que sucedan los errores, sin intervenir en su solución, excepto cuando no se cumplen los estándades (Sosik y Jung, 2018). La DEP constituye la conducta de liderazgo altamente evitativo y, según los estudios de Lee y Jensen (2014), se relaciona directamente con comportamientos descorteses en el trabajo e indirectamente con percepciones de equidad.

Liderazgo laissez-faire (LF). Referido a la presencia de conductas que no son consideradas como liderazgo. Sosik y Jung (2018) lo definen como propio de aquellos líderes que evitan involucrarse en las tareas y en los problemas, son indiferentes, reacios a tomar decisiones, apáticos y distraídos, todo lo cual indica que no existe relación entre el líder y las personas.

Satisfacción, efectividad y esfuerzo extra (S, E, EE). Son variables relacionadas con el impacto del liderazgo y miden la incidencia de las conductas del líder. La primera noción está relacionada con la forma como el líder logra llenar las expectativas y necesidades de los seguidores. La efectividad se refiere al alcance de resultados óptimos, producto del buen manejo de los recursos y procesos. Finalmente, con el esfuerzo extra 
se logra que el equipo alcance los resultados, metas y objetivos y se superen las expectativas.

Multifactor Leadership Questionnaire (MLO). Es el instrumento validado para medir las tipologías de liderazgo en el modelo LRF. De acuerdo con Avolio et al. (1999), la base teórica de este cuestionario comenzó con los estudios de Burns (1978) sobre LET. La mayoría de trabajos sobre LET han utilizado este instrumento, el cual ha tenido sinnúmero de observaciones; por ejemplo, Hater y Bass (1988, en Avolio et al., 1999) lo modifican, al proponer que la conducta de gestión por excepción podría ser pasiva o activa.

En conclusión, el FRL ha sido uno de los modelos más utilizados en el estudio del liderazgo. Es un modelo compuesto de distintas variables que examinan diversas tipologías de liderazgo. Comenzó a utilizarse antes de 1985 y sigue vigente hasta nuestros días. Respecto de su utilización en el contexto educativo de Colombia, tenemos los estudios de Jaime (2016) y de Jaime, Cáceres e Hinojosa (2018), autores que utilizan el MLQ aplicado a docentes y directores de programas tecnológicos de la Universidad Cooperativa de Colombia. En estas investigaciones se estudia el liderazgo de profesores y directores mediante la autopercepción y la percepción de los estudiantes (Jaime, 2016).

\section{Metodología}

El estudio es de tipo cualitativo y exploratoriodescriptivo, basado en la revisión documental, con la finalidad de detectar trabajos empíricos y conceptos clave (Hernández, Fernández y Baptista, 2014), con la participación de expertos. Se llevó a cabo en cuatro etapas:1) de revisión bibliográfica, 2) de estudio y análisis de propuestas para desarrollo del modelo, 3 ) de estudio exploratorio y 4) de validación del instrumento.

\section{Etapa 1. Revisión bibliográfica}

Tuvo como propósito indagar por los estudios y teorías acerca del tema de investigación. Para tal fin, se acudió a los tesauros y a la técnica de cartografía social. Se tomaron como unidades de análisis: liderazgo, teorías o enfoques de liderazgo, liderazgo en la educación. Lo anterior permitió el acceso a información y la consulta de estudios en diferentes plataformas de información y libros. El criterio de selección de los artículos y libros tuvo en cuenta que estuvieran lo más cercanos posible al tema. Finalmente, se procedió a la lectura de los documentos seleccionados y a extraer las ideas relevantes que tuvieran relación con los asuntos definidos en la investigación.

\section{Etapa 2. Estudio y análisis de propuestas para desarrollo del modelo}

De acuerdo con los hallazgos encontrados en la etapa 1, se procedió al estudio y adaptación para MELED. Se analizaron varias opciones: la primera, adoptar la malla gerencial de Robert Blake y Jane Mouton, la cual combina en una matriz el enfoque o dimensión implicados en los procesos y el enfoque o dimensión en las tareas (Sánchez, 2008; Giraldo y Naranjo, 2014), además, permite identificar diferentes tipos de liderazgo: sinérgico, tecnicista, sociable, indiferente (Sánchez, 2008). La segunda opción fue trabajar el LT con sus cuatro componentes (IA, IC, MI, EI); como tercera opción, se contempló el modelo FRL, como producto de los ajustes realizados, de acuerdo con las observaciones de los expertos.

\section{Etapa 3. Estudio exploratorio}

Dado que los modelos descritos en la etapa 2 requerían ser aprobados y validados por expertos, se llevó a cabo un estudio exploratorio, por sugerencia de uno de ellos. Dicho estudio tuvo como finalidad reconocer algunas variables de la población y del contexto donde se realiza la investigación. Entre las variables analizadas están: número de instituciones educativas rurales en el municipio; número de docentes y estudiantes por institución; indicadores escolares, como cobertura, deserción en los tres últimos años e infraestructura escolar; identificación de las sedes educativas; vías de acceso; problemas de contexto escolar: social, económico y seguridad. 
Asimismo, variables relacionadas con la población docente: tiempo laborado en la institución, años de experiencia, formación académica y conocimiento de algunos tipos de liderazgo educativo, así como su disposición para participar en el estudio.

\section{Etapa 4. Validación del instrumento}

Para la adopción del modelo final, los instrumentos asociados fueron sometidos a procesos de evaluación por parte de expertos (tres internacionales y dos nacionales). La primera opción: modelo relacionado con la malla gerencial del liderazgo, fue descartada, por considerarse complejo medir los efectos de las tipologías de liderazgo identificadas en el modelo. La segunda opción: modelo basado en el LET, fue presentada a los expertos, quienes hicieron recomendaciones y sugerencias, las cuales fueron estudiadas y adoptadas.

Uno de los expertos nacionales encontró algunas inconsistencias entre el modelo propuesto con su respectivo cuestionario y el plantemiento del problema, mientras que el segundo enfatizó en los objetivos de la investigación y el instrumento. La experta internacional realizó algunas recomendaciones: delimitación del problemas, enfatizar en los antecedentes del problema, estudio exploratorio, mejorar la operación de las variables, mientras que el segundo experto internacional recomendó la inclusión de otras variables y tener en cuenta los estudios realizados por Vega y Zabala (2004).

A partir de las recomendaciones de los expertos, se procedió a reformular el modelo, producto del cual nace la tercera opción, consistente en la adapatación del modelo FRL. Al FRL adaptado como meLED se le asoció como instrumento la versión MLO forma $5 x$ utilizada por Vega y Zabala (2004), pero adaptada. Las preguntas o ítems de este cuestionario se revisaron individualmente y se ajustaron a un lenguaje acorde a la relación docente-estudiante, a las interacciones en los procesos de educativos, al contexto escolar, al modelo pedagógico de las instituciones participantes y, en general, a los procesos educativos.
El cuestionario se ajustó en la versión enviada a docentes y estudiantes. Las dos versiones ajustadas fueron revisadas nuevamente por la experta internacional, en este caso, la directora de tesis origen del presente artículo. Una vez la experta dio el visto bueno, se hicieron simulacros con estudiantes y docentes. Estos simulacros, entre otros elementos, buscaban verificar: nivel de comprensión de cada una de las preguntas por parte de los encuestados (docentes y estudiantes de las instituciones rurales objeto de estudio); tiempo en responder el cuestionario, aceptación para participar y posibles sugerencias. A partir del simulacro, se concluyó que las preguntas eran claras y, además, se podían aplicar a estudiantes de grado sexto en adelante. Algunos docentes expresaron que el cuestionario resultaba largo, otros dijeron que el procesamiento y análisis resultarían bastante complejos o que, aunque se miden muchas variables, igual resultaría fácil de entender y ser aplicado.

\section{Resultados}

Producto de esta investigación, describimos tres tipos de resultados. El primero, fruto del estudio exploratorio, nos da una radiografía del contexto de las instituciones participantes; el segundo contiene el Modelo de Estudio del Liderazgo Educativo Docente (meled); y el tercero, el análisis de confiabilidad y correlacional del modelo, compuesto por variables de primer y segundo orden. Cada variable queda definida conceptualmente en términos del contexto educativo y la relación docente-estudiante. Del mismo modo, las variables se definen operacionalmente. Por último, al modelo se le asocian dos tipos de cuestionario de liderazgo educativo docente (CLED): una versión para docentes y otra para estudiantes.

\section{Resultado 1. Caracteristicas del contexto institucional}

El municipio de Tuluá cuenta con 18 instituciones educativas oficiales. De ellas, 9 son urbanas 
y 9 rurales, y de estas, 6 están ubicadas en media y alta montaña. En la Tabla 2 se describe el número de estudiantes y docentes de estas instituciones. $\mathrm{El}$ estudio se realiza en las instituciones educativas Monteloro (IE1) y San Rafael (IE2). La primera tiene una población de 193 estudiantes y 16 docentes y la segunda cuenta con 495 educandos y 34 docentes. Los indicadores escolares de cobertura y deserción presentan un comportamiento positivo, pues en los tres últimos años ambas instituciones han mantenido o mejorado la cobetura, y la deserción escolar es mínima, comparada con la del municipio, incluso en épocas de estudio en casa por pandemia.

La IE1 está conformada por 6 sedes educativas (2 de ellas cerradas temporalmente) y la IE2 tiene 15. Dichas sedes están ubicadas en zona dispersa y de difícil acceso, en su mayoría, con grandes problemas de tipo social y económico. La disputa por el territorio ocasionada por grupos al margen de la ley ha ocasio- nado desplazamiento, pobreza y muerte, aunque la desmovilización de dichos grupos permitió que esta situación cambiara. La mayoría de las sedes educativas funcionan con la metodología de la Escuela Nueva, para instituciones unitarias multigrado.

Respecto al personal docente, el 97\% corresponde a licenciados en educación y el 30\% cuenta con algún estudio de posgrado (especialización, maestría o doctorado). El 9o\% tiene 50 más años de experiencia en educación rural, solo el 10\% tiene menos de 5 años de experiencia y el $55 \%$ son mujeres y el $45 \%$ hombres. Al interactuar con estos docentes respecto a su labor, se puede evidenciar gran aprecio por la profesión docente y vocación por el ejercicio pedagógico; asimismo, se observa gran estima por los estudiantes y una relación armónica con los estudiantes y padres de familia. Ello también se corroboró al establecer contacto con unos y otros en las diferentes veredas que se visitaron.

Tabla 2. Población estudiantil por IE: Tuluá, 2021

\begin{tabular}{|c|c|c|c|}
\hline Institución educativa & Ubicación & No. de docentes & Matricula \\
\hline Gimnasio del Pacífico & Urbana & 76 & 2036 \\
\hline Monteloro & Rural media y alta & 16 & 193 \\
\hline San Juan de Barragán & Rural media y alta & 23 & 335 \\
\hline San Rafael & Rural media y alta & 34 & 495 \\
\hline Aguaclara & Rural plana & 78 & 1762 \\
\hline Alfonso López Pumarejo & Urbana & 96 & 2645 \\
\hline Altorrocio & Rural media y alta & 13 & 146 \\
\hline Corazón del Valle & Urbana & 79 & 2054 \\
\hline Jovita Santa Coloma & Rural plana & 26 & 509 \\
\hline Juan María Céspedes & Urbana & 88 & 2545 \\
\hline Julia Restrepo & Urbana & 88 & 2761 \\
\hline Julio César Zuluaga & Rural plana & 21 & 421 \\
\hline Moralia & Rural media y alta & 20 & 322 \\
\hline María Antonia Ruiz & Urbana & 72 & 1971 \\
\hline Moderna & Urbana & 91 & 2634 \\
\hline Técnico Industrial & Urbana & 60 & 1360 \\
\hline La Marina & Rural media y alta & 40 & 810 \\
\hline Occidente & Urbana & 104 & 2808 \\
\hline Total & & 1024 & 25.807 \\
\hline
\end{tabular}

Fuente: Secretaría de Educación, basado en el Sistema de Matrícula Estudiantil de Educación Básica y Media (Simat), 23 de marzo de 2021. 
En el tema de infraestructura y cobertura en comunicación, las instituciones presentan ciertas dificultades. La sede central de la IE2 no tiene una sede acorde con la cantidad de estudiantes, los estudiantes de básica primaria deben recibir clases en casas alquiladas y de manera similar ocurre con otras sedes unitarias. En el caso de la IE1, dos de sus sedes funcionan en casas alquiladas o prestadas. En lo que respecta a medios de comunicación (internet, llamadas de voz), se presentan problemas de falta de cobetura o ausencia de conectividad.

\section{Resultado 2. Modelo de Estudio del Liderazgo Educativo Docente}

En la Figura 1 se presentan los elementos del modelo MELED. Las variables de primer nivel corresponden a estilos o tipologías de liderazgo educativo: transformacional (LET), transaccional (LES) y correctivo evitador (LECE). Los elementos de segundo nivel corresponden a las conductas de los docentes, y a cada tipología de liderazgo le corresponde un grupo de conductas. El tercer elemento del modelo corresponde a los efectos o incidencia de los estilos de liderazgo, que se traducen en innovación educativa y transformación social.

Al LET le corresponden las conductas CIA, CIC, El y MI. Estas conductas se espera que redunden en procesos de innovación educacional, pues los hallazgos muestran una relación entre liderazgo e innovación y calidad educativa (Leal, Albornoz, Rojas, 2016; Blanco, 2017). Para el caso del LES, las conductas asociadas son Cl y RC. Por último, el LECE está conformado por las conductas DEA, DEP y LF.

\section{Conceptualización de los elementos del modelo}

En seguida desarrollamos la conceptualización teórica de cada uno de los elementos del modelo, que se construye con base en las definiciones de las variables o factores del FRL y la adaptación que de este se hace al contexto escolar.

\section{LIDERAZGO EDUCATIVO TRANSFORMACIONAL (LET)}

Un docente tranformacional genera procesos de transformación social y tiene hablidades para encauzar a sus estudiantes hacia el desarrollo de sus potencialidades y habilidades, al estimular el aprendizaje y establecer desafíos pedagógicos complejos. Al ser fuente de inspiración para sus estudiantes, estos lo imitan y quieren ser como él; es éticamente integral y poderoso, e intelectualmente inquieto. En conclusión, el LET se ve reflejado en conductas como el ser carismático, la capacidad para estimular intelectualmente a los estudiantes y ser fuente de inspiración y transformación social.

De acuerdo con los resultados en otros campos, para el sector educativo el LET puede conducir a resultados positivos y a la mejora de indicadores escolares. Este tipo de liderazgo mejora la participación de estudiantes, padres de familia y cuidadores, al sentirse influenciados, motivados e incluidos en las metas institucionales. Incide positivamente en las condiciones de satisfacción ante los procesos escolares y permite que los estudiantes, producto de una alta motivación y estimulación intelectual, se esfuercen en alcanzar los resultados deseados. Por último, el LET promueve el autoliderazgo en los estudiantes, lo que, según Melo y Mendonça (2020), permite que estos no aplacen las actividades académicas y dediquen más tiempo a lo importante. Como resultado de este último elemento, se reduce la deserción escolar y mejora la asistencia educativa.

De otra manera, al ser la educación el eje central de la sociedad, el LET se convierte en una herramienta de transformación social, debido a que promueve la innovación (Ryan y Tipu, 2013) y contribuye con un clima de participación y de metas compartidas. Esto conduce a lo que el Ministerio de Educación Nacional (2008) ha definido como mejoramiento continuo, pues el liderazgo incrementa el compromiso y reduce las tensiones, la hostilidad y el estrés (Trógolo, Pereyra y Sponton, 2013). Asimismo, el LET aporta igredientes importantes para que las 
instituciones educativas se conviertan en lo que Bolívar (2007) señala como unidades básicas de formación e innovación y organizaciones que aprenden. Todo ello redunda en la tranformación social, pues la educación es el motor del desarrollo social.

- Carisma con influencia idealizada conductual. El docente con este carisma tiene claros los objetivos, propósitos, fines y logros de la clase, del área que orienta, así como los institucionales, que son compartidos con los estudiantes. Se establecen metas claras, con las cuales se identifican todos y en las cuales trabajan para alcanzarlas. Con su comportamiento, el docente se convierte en modelo a seguir y a ser imitado por los estudiantes. El docente conoce sus necesidades y comparte riesgos con ellos. Asimismo, es admirado y respetado y tiene su confianza. Esta conducta está relacionada con la capacidad del líder para influir positivamente en los estudiantes, lo que le permite ser una fuente de inspiración para ellos, quienes lo admiran y consideran atractivas y oportunas sus orientaciones.

- Carisma con influencia idealizada atribuida. Como la anterior, acá se trata de los atributos que observa el estudiante en el docente o le son atribuidos.

- Estimulación intelectual. El docente orienta a sus estudiantes a contrarrestar las barreras y obstáculos del contexto. Asimismo, les propone actividades y ejercicios con diferentes niveles de profundidad o dificultad y los orienta de manera adecuada en sus procesos. Los docentes que estimulan intelectualmente a sus estudiantes son capaces de guiar cambios positivos, potenciar las capacidades y estimular el esfuerzo, el aprendizaje y la creatividad. De otro lado, la estimulación intelectual también está relacionada con las habilidades del docente para innovar en la enseñanza y en la didáctica. Esta conducta promueve la innovación educacional, la autonomía y la independencia de los estudiantes.

- Motivación inspiracional. El docente favorece la participación y el trabajo colaborativo de todos los estudiantes, que trabajan por el bienestar del grupo. A docentes empoderados estudiantes empoderados. Esta conducta hace que el docente genere entusiasmo y los estudiantes encuentren motivos y razones para aprender o contribuir con sus procesos de formación, realizar las tareas y construir sus proyectos.

LIDERAZGO EDUCATIVO TRANSACCIONAL (LES)

El docente transaccional se mueve entre el liderazgo transformacional y el liderazgo correctivo evitador; es decir, puede tener conductas de uno, del otro o de los dos. Por otro lado, el docente transaccional está dispuesto a establecer acuerdos con sus estudiantes, reglas de juego y acciones de mejora. Los acuerdos pueden ir desde las normas de convivencia en el aula de clases, que sean claramente establecidas, hasta establecer las recompensas por el cumplimiento en la realización de tareas. Las recompensas pueden darse por llegar temprano, por colaborar, por hacer parte de un equipo, por representar al grupo o a la institución, entre otras.

El LES permite que los docentes desplieguen conductas relacionadas con la consideración individualizada de los estudiantes, lo cual conlleva atenderlos según sus necesidades, debilidades y fortalezas. De la misma manera, despliega conductas y aptitudes relacionadas con la recompensa contigente. Por su parte, el asumir en su comportamiento laboral en el aula de clases una actitud de recompensa contigente no es ni bueno ni malo para los procesos educativos; todo está supeditado a las necesidades del contexto, a las condiciones escolares y a la realidad estudiantil.

- Consideración individualizada. El docente conoce y atiende las necesidades de cada estudiante. Realiza grandes esfuerzos para mejorar las 
necesidades de sus estudiantes y potenciar sus habilidades. Posee capacidades para atender a cada estudiante, de acuerdo con el contexto y las particularidades personales. Atiende los diferentes ritmos de aprendizaje del educando, se interesa por la asistencia a clases, reporta de manera oportuna cualquier anomalía que tenga el educando en lo personal o en el proceso académico, e involucra a las familias en el proceso de formación estudiantil.

- Recompensa contingente. El docente explica todo lo que espera de cada estudiante y de la clase. Asimismo, presenta los estímulos y dádivas que recibirán si alcanzan los desempeños esperados. Premia el buen desempeño del estudiante: "Quien termine primero, puede ir a descanso", "quien saque 5 en este trabajo no presentará examen final", "a quienes presenten todas las consultas, se les adiciona o, 5 en la nota final". Su discurso pedagógico se caracteriza por enfatizar siempre en el premio o en el castigo, si hay falta.

LIDERAZGO EDUCATIVO CORRECTIVO EVITADOR (LECE)

El docente evita inmiscuirse en los asuntos o problemas de los estudiantes y los conoce poco. Pocas veces interviene en los problemas personales y familiares y, si lo hace, será por una situación o condición extrema, una obligación extra o porque la circunstancia lo amerita. No establece normas de disciplina o convivencia claras. De igual manera, pocas veces hace seguimiento al desempeño de los estudiantes $y$, cuando lo hace, es solo porque hay factores externos que lo obliguen. En ocasiones, el accionar docente puede ser coherente con el contexto de los estudiantes y el modelo educativo de la institución educativa.

- Docencia por excepción activa (DEA). Los docentes con esta conducta se dedican a inspeccionar la realización de tareas de los estudiantes y buscan y corrigen los errores. En otras palabras, centran su esfuerzo en verificar si los estudian- tes hicieron la tarea, identificar errores y explicarles cómo debe hacerse.

- $\quad$ Docencia por excepción pasiva (DEP). El docente, como director de grupo, o simplemente en su rol de docente de aula, se muestra pasivo. No toma acciones correctivas ante las fallas $y$, si lo hace, es solo después de que estas sucedieron y las dificultades se han tornado serias o se han agravado. Realiza las acciones correctivas cuando se las piden. Es típico de un docente al que el directivo debe llamarle la atención permanentemente.

- Liderazgo laissez-faire (LF). Referido a la presencia de conductas que no son consideradas como liderazgo. Docente que no cumple con sulabor.

Resultados (se, eE, EeE)

Como resultados se tienen: Satisfacción escolar (SE): el docente logra las expectativas de los estudiantes, a quienes les agrada trabajar en clase, y aumenta el deseo de ir a clases y alcanzar los resultados deseados. Efectividad escolar (EE): los docentes alcanzan resultados óptimos y los educandos asumen el rol que les corresponde, de manera autónoma. Esfuerzo extraescolar (EEE): los estudiantes desean ir al colegio, a pesar de las dificultades, y realizan las tareas para alcanzar sus propias metas y las de la institución. Se generan así procesos de innovación educacional y transformación social.

\section{Operación de las variables en el modelo}

Para la medición o evaluación de las 15 variables que componen el modelo MELED, en el cuestionario de liderazgo educativo docente (CLED), tanto para estudiantes como para docentes, cada pregunta está dirigida a medir uno u otro elemento o variable del modelo. En la Tabla 3 se relacionan dichos elementos o variables y el número o los números de las preguntas que les corresponden en el cuestionario. 
Figura 1. Modelo de Estudio de Liderazgo Educativo Docente
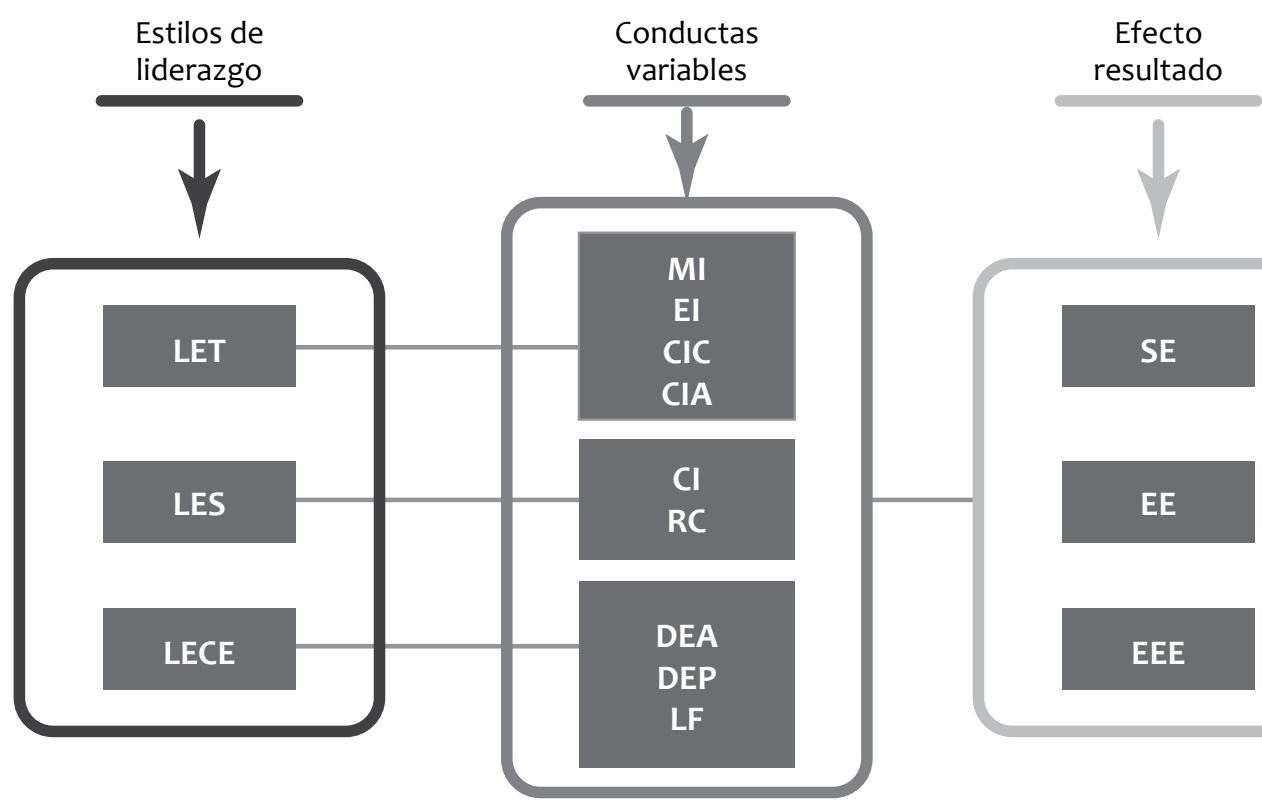

SE

EE

Fuete: elaboración propia.

Tabla 3. Medición de las variables en el MELED

\begin{tabular}{|c|c|}
\hline Variables & Preguntas relacionadas en el cuestionario \\
\hline \multicolumn{2}{|r|}{ LET } \\
\hline $\mathrm{CIC}$ & $6,14,23,34,46,57,61,71$ \\
\hline $\mathrm{CIA}$ & $10,18,21,25,47,58,80$ \\
\hline El & $2,8,30,32,49,60,69,73$ \\
\hline MI & $9,13,26,36,48,59,72,81$ \\
\hline \multicolumn{2}{|r|}{ LES } \\
\hline $\mathrm{Cl}$ & $15,19,29,31,50,62,74,77$ \\
\hline$C R$ & $1,11,16,35,51,63$ \\
\hline \multicolumn{2}{|r|}{ LECE } \\
\hline DEA & $4,22,24,27,52,64$ \\
\hline DEP & $3,12,17,20,53,65$ \\
\hline LF & $5,7,28,33,54,66,75,78$ \\
\hline SE & $38,41,70,76,82$ \\
\hline $\mathrm{EE}$ & $37,40,43,45,56,68$ \\
\hline EEE & $39,42,44,55,67,79$ \\
\hline
\end{tabular}

Fuente: elaboración propia, con base en Vega y Zabala (2004). 


\section{Resultado 3. Confiabilidad y correlacionalidad del MELED}

El anáilis de confiabilidad se lleva a cabo mediante el alfa de Cronbach, índice cuyo valor oscila entre o y 1 , de modo que mientras más cerca esté de 1 mayor confiabilidad tendrá el instrumento. Pestana y Gageiro (2000, en Santos, 2016) describen la siguiente escala alfa de Cronbach para la confiabilidad del instrumento: entre o,9 y 1: confiablidad excelente; entre 0,8 y 0,9 : muy buena confiabilidad; entre 0,7 y 0,8: confiabilidad buena; entre 0,6 y 0,7: confiabilidad relativamente baja; entre 0,5 y o,6: el instrumento necesita revisión; y si es inferior a 0,5, es considerado bajo, poco creíble.

Tabla 4. Análisis de confiabilidad: alfa de Cronbach

\begin{tabular}{|c|c|}
\hline IE1 & IE2 \\
\hline 0,929 & 0,913 \\
\hline
\end{tabular}

Fuente: elaboración propia.

Para ambas instituciones tenemos un alfa de Cronbach superior a 0,9 (Tabla 4). Lo anterior signi- fica que el modelo y su cuestionario presentan una confiabilidad bastante alta, considerada excelente.

Para el análisis de correlacionalidad usamos el coeficiente de correlación de Pearson, que oscila entre -1 y 1 , donde valores cercanos a 1 indican un nivel de relación o correlación positivo muy significativo, mientras que los cercanos a -1 indican una correlación negativa o inversa muy alta, y los cercanos a o implican baja correlación. Se realiza análisis correlacional con pregunta variable de primer orden con variable de segundo orden y variable de primer y segundo orden con variables de resultados. Se observa que cada una de las preguntas presenta una correlación positiva y significativa (con nivel de significancia del 1\%) con la variable correspondiente.

En la Figura 2 se describen los índices de correlación entre las variables del modelo. Vemos que existe una correlación fuerte y significativa entre variables de segundo orden y la correspondiente variable de primer grado (estilos de liderazgo), excepto entre el LECE y la variable DEA. Es de aclarar que la variable DEA se comporta como una conducta po-

Figura 2. Correlación entre variables IE1

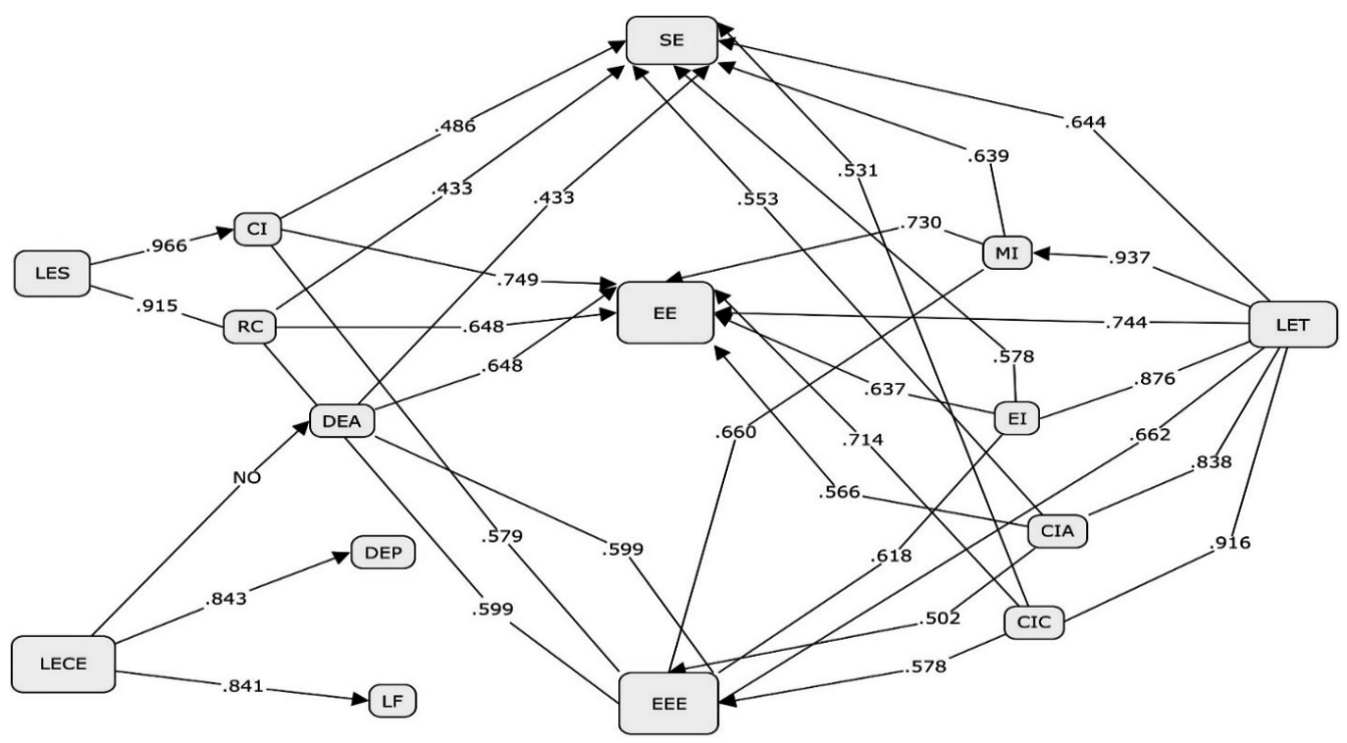

Fuente: elaboración propia. 
sitiva en la docencia, mientras las demás variables del LECE son conductas pasivas o de ausencia de liderazgo. También se aclara que todas las preguntas de la variable DEA se correlacionaron con dicha variable. Por último, la correlación con las variables de resultados también es significativa; por ejemplo, la correlación entre la variable MI y la EE es de o,730, lo cual indica un alto nivel de asociación o relación entre la motivación inspiracional de los docentes y el esfuerzo extra de los educandos. Para la IE2 los índices de correlación son similares a los de la IE1.

\section{Consideraciones finales y conclusiones}

Del estudio se derivan algunas consideraciones y conclusiones que son importantes de analizar. Primero, la educación rural es un componente del sistema educativo que merece especial atención. A los problemas comunes en el sector educativo colombiano en la ruralidad se les suman problemáticas de contexto, como la falta de cobertura de internet o llamadas de voz, sedes ubicadas en zonas de difícil acceso, precariedad de las vías de acceso y problemas de inseguridad. También es de resaltar el problema de movilidad de las poblaciones. No obstante, el proceso educativo en estas instituciones es positivo, con buena cobertura educativa, baja deserción y repitencia, lo cual se puede atribuir a la buena labor de los docentes y a su liderazgo educativo.

Segundo, se alcanzaron los objetivos de la investigación. El MELED que se propone permite estudiar conductas, rasgos y actitudes que desbordan diversos tipos de liderazgo del docente de aula. Dichas conductas y atributos podrán ser identificados y valorados por el docente y por los estudiantes a través del instrumento, que ha sido previamente avalado. El FRL adaptado resulta ser una herramienta propicia para estudiar el liderazgo docente en contextos educativos, lo que indica un aporte significativo de este estudio empírico. Por tratarse el FRL de un modelo multiliderazgo, puede incluir elementos de liderazgo compartido. En cuanto a los elementos de Maureira, Moforte y González (2014) y Maureira, Garay y López (2016), muestran la eficacia del liderazgo distribudio en entornos educativos.

Tercero, el MELED es funcional, consistente y confiable. Los estadísticos de confiabilidad son altos y consistentes. De la misma manera, la significativa correlación entre variables del cuestionario sugiere que las preguntas son adecuadas para medirlas. De igual manera, la correlación entre variables de primer y segundo orden sugiere una nivel de asociación positivo y óptimo.

Cuarto, a partir del Meled se pueden llevar a cabo estudios de liderazgo docente en las instituciones educativas, con base en los cuales generar procesos de capacitación y formación docente en liderazgo. Dicha formación puede incidir en procesos de empoderamiento y contribuir a los fines denominados por Salazar y Tobón ( 2018) como propios de la sociedad del conocimiento. De otro lado, las conductas de liderazgo podrán incidir positivamente en la mejora de las condiciones en zonas como en las que se llevó a cabo esta investigación y, en general, en regiones como América Latina, caracterizada por Krüger (2019) como una región con baja inclusión. 


\section{Referencias}

Arévalo, V. A., Tikhomirova, A., Trejo Toriz, A. C. y García Ramírez, J. M. (2015). Liderazgo transaccional vs. Liderazgo transformacional. REiDoCrea. Revista Electrónica de Investigación y Docencia Creativa, 4, 24-27 Recuperado de: https://digibug.ugr.es/bitstream/handle/10481/34629/AlmironArevalo_V4_ Art4. pdf? sequence $=6 \&$ is Allowed $=y$

Avendaño Castro, W. R., Paz Montes, L. S. y Parada-Trujillo, A. E. (2016). Estudio de los factores de calidad educativa en diferentes instituciones educativas de Cúcuta. Investigación \& Desarrollo, 24(2), 329-354. DOI: https://doi.org/10.14482/indes.24.2.8031

Avolio, B., Bass, B.y Jung, D. (1999). Re-examining the components of transformational and transactional leadership using the Multifactor Leadership Questionnaire. Journal of Occupational and Organizational Psychology, 441-462. DOI: https://doi.org/10.1348/096317999166789

Barbosa Gómez A. M., Briceño Vargas C. A., Gómez Rincón, Y. M., Lizarazo Sierra, M. M. y Maguin Martínez, T. (2018). El ejercicio del liderazgo docente en Colombia: voces de los maestros. [Tesis maestría], Universidad de La Sabana, Chía, Colombia. Recuperado de: http://hdl.handle.net/10818/33433

Bass, B. (1990). From transactional to transformational leadership: Learning to share the vision. Organizational Dynamics, 18(3), 19-31. DOI: https://doi.org/10.1016/0090-2616(90)90061-S

Bass, B. M. (1985). Leadership and performance beyond expectation. Nueva York: Free Press.

Bedoya Dorado, C., Murillo, V. G. y González Campos, C. H. (2020). Competencias directivas en la gestión universitaria en Colombia. Educación y Educadores, 23(4), 685-708. DOI: https://doi.org/10.5294/edu.2020.23.4.7

Bolívar, G. (2007). Los centros educativos como organizaciones que aprenden. Promesas y realidades. Madrid: La Muralla.

Blanco Figueredo, L. L. (2017). Liderazgo e innovaciones pedagógicas como puntos de partida para lograr una educación de calidad. Revista Cientifica Eduser, 4(1), 1-15. Recuperado de: http://revistas.ucv.edu.pe/index. php/EDUSER/issue/view/1902.038

Burns, J. M. (1978), Leadership. Nueva York: Harper y Row.

Castillo, F. A. y Hallinger, P. (2018). Systematic review of research on educational leadership and management in Latin America, 1991-2017. Educational Management Administration \& Leadership, 46(2), 207-225. Recuperado de: https://doi.org/10.1177/1741143217745882

Castro Solano, A., Lupano Perugini, M., Benatuil, D. y Nader, M. (2007). Teoría y evolución del liderazgo. Buenos Aires: Paidós.

Contreras Torres, F. y Barbosa Ramírez, D. (2013). Del liderazgo transaccional al liderazgo transformacional: implicaciones. Revista Virtual Universidad Católica del Norte, 39(2), 151-164. Recuperado de: https://repository. urosario.edu.co/handle/10336/27482 
Daft, R. L. (2006). The experience ofleadership. Mason: Cengage Learning.

Durán Gamba, M. G. y Castañeda Zapata, D. I. (2015). Relação entre liderança transformacional e transacional com a conduta de compartilhar conhecimentos em duas empresas de serviços. Acta Colombiana de Psicología, 18(1), 135-147. DOI: https://doi.org/10.14718/ACP.2015.18.1.13

Escribano Hervis, E. (2018). El desempeño del docente como factor asociado a la calidad educativa en América Latina. Revista Educación, 42(2), 717-739. DOI: https://doi.org/10.15517/revedu.v42i2.27033

Flessa, J., Bramwell, D., Fernandez, M. y Weinstein, J. (2017). School leadership in Latin America 2000-2016. Educational Management Administration y Leadership, 46(2), 182-206. DOI: https://doi.org/10.1177/1741143217717277

García Solarte, M. (2015). Formulación de un modelo de liderazgo desde las teorías de las organizaciones. Entramado, 11(1), 60-79. DOI: https://doi.org/10.18041/entramado.2015V11n1.21111

Giraldo, D. y Naranjo Agudelo, J. A. (2014). Liderazgo: desarrollo del concepto, evolución y tendencias. Estudio Mográfico, Universidad del Rosario, Bogotá, Colombia. Recuperado de: https://repository.urosario.edu.co/ handle/10336/8672

Gómez Rada, C. (2002). Liderazgo: conceptos, teorías y hallazgos relevantes. Cuadernos Hispoamericanos de Psicología, 2(2), 61-77. Recuperado de: http://www.elmayorportaldegerencia.com/Libros/ Liderazgo/\%5BPD\%5D\%20Libros\%20-\%2oLiderazgo.pdf

Hernández-Castilla, R., Murillo, F. J. e Hidalgo Farran, N. (2017). Lecciones aprendidas del estudio del liderazgo escolar exitoso. Los casos de España en el Proyecto Internacional ISSPP. Revista de Investigación Educativa, 35(2), 499-518. DOI: https://doi.org/10.6018/rie.35.2.279241

Hernández Sampiero, R., Fernández Collado, C. y Baptista Lucio, M. D. (2014). Metodología de la investigación. México: McGraw-Hill.

Horn, A. y Marfán, J. (2010). Relation between educational leadership and school achievement: Review of the research in Chile. Psicoperspectivas. Individuo y Sociedad, 9(2), 82-104. Recuperado de: https://www.psicoperspectivas.cl/index.php/psicoperspectivas/article/view/116

Hurtado de Barrera, J. (2019). Un acercamiento sintagmático al concepto de liderazgo. Desarrollo Gerencial, 11(1), 190-208. DOI: https://doi.org/10.17081/dege.11.1.3465

Ibáñez Delgado, N. (2011). Aprendizaje-enseñanza: mejora a partir de la interacción de los actores. Educación y Educadores, 14(3), 457-474. DOI: https://doi.org/10.5294/edu.2011.14.3.1

Jaime Cuadros, M. del P. (2016). Estudio del liderazgo de docentes y directivos en programas técnicos-tecnológicos de la Universidad Cooperativa de Colombia, de Bucaramanga, Colombia. [Disertación doctoral], Universidad de Granada, Departamento de Didáctica y Organización Escolar. Recuperado de: https://digibug.ugr. es/handle/10481/40898 
Jaime Cuadros, M. del P., Cáceres, R. e Hinojo Lucena, F. J. (2018). Analysis of leadership styles developed by teachers and administrators in technical-technological programs: the case of the Cooperative University of Colombia. International Journal of Leadership in Education, 21(1), 1-16. DOI: https://doi.org/10.1080/1360312 4.2016.1172734

Jensen, U. T., Andersen, L. B., Bro, L. L., Bøllingtoft, A., Eriksen, T. L. M., Holten, A. L. y Würtz, A. (2019). Conceptualizing and measuring transformational and transactional leadership. Administration \& Society, 51(1), 3-33. DOI: https://doi.org/10.1177/0095399716667157

Jiménez Díaz, J. F. (2008). Enfoque sociológico para el estudio del liderazgo político. Barataria. Revista Castellano-Manchega de Ciencias Sociales, 9, 189-203. Recuperado https://doi.org/10.20932/barataria.voig.186

Krüger, N. (2019). La segregación por nivel socioeconómico como dimensión de la exclusión educativa: 15 años de evolución en América Latina. Archivos Analíticos de Políticas Educativas, 27(8), 1-37. DOI: https://doi. org/10.14507/epaa.27.3577

Leal Soto, F., Albornoz Hernández, M. y Rojas Parada, M. I. (2016). Liderazgo directivo y condiciones para la innovación en escuelas chilenas: el que nada hace, nada teme. Estudios Pedagógicos (Valdivia), 42(2), 193-205. DOI: https://doi.org/10.4067/S0718-07052016000200011

Lee, J. y Jensen, J. M. (2014). The effects of active constructive and passive corrective leadership on workplace incivility and the mediating role of fairness perceptions. Group \& Organization Management, 39(4), 416443. DOI: https://doi.org/10.1177/1059601114543182

Leithwood, K. y Mascall, B. (2008). Collective leadership effects on student achievement. Educational Administration Quarterly, 44(4), 529-561. DOI: https://doi.org/10.1177/0013161X08321221

Leithwood, L. y Jantzi, D. (2000). The effects of transformation leadership on student engagement with school. Journal of Educational Administration, 39(2), 112-129. DOI: https://doi.org/10.1108/09578230010320064

Maureira Cabrera, O., Garay Oñate, S. y López Alfaro, P. (2016). Reconfigurando el sentido del liderazgo en organizaciones escolares contemporáneas: la perspectiva del liderazgo distribuido. Revista Complutense de Educación, 27(2), 689-706. DOI: https://doi.org/10.5209/rev_RCED.2016.v27.n2.47079

Maureira, O., Moforte, C. y González, G. (2014). Más liderazgo distribuido y menos liderazgo directivo: Nuevas perspectivas para caracterizar procesos de influencia en los centros escolares. Perfiles Educativos, 36(146), 134-153. DOI: https://doi.org/10.1016/S0185-2698(14)70132-1

Melo, T. G. y Mendonça, H. (2020). Academic procrastination: relationships with support from the environment and self-leadership. Paidéia, 30(eз33), 1-9. DOI: https://doi.org/10.1590/1982-4327ezo38

Mendoza Torres, M. y Ortiz Riaga, C. (2006). El liderazgo transformacional: dimensiones e impacto. Investigación y Reflexión, XIV(1), 118-134.

McCleskey, J. A. (2014). Situational, transformational, and transactional leadership and leadership development. Journal of Business Studies Quarterly, 5(4), 117-130. 
Ministerio de Educación Nacional (2008). Guía para el mejoramiento institucional de la autoevaluación al plan de mejoramiento. Serie Guías, No. 34. Recuperado de: https://www.mineducacion.gov.co/1621/article-177745.html

Muenjohn, N. (2008). Evaluating the structural validity of the Multifactor Leadership Questionnaire (MLO), capturing the leadership factors of transformational-transactional leadership. Contemporary Management Research, 4(1), 3-14. DOI: https://doi.org/10.7903/cmr.704

Munroe, M. (2014). The power character in leadership: how values, morals, ethics, and principles affecst leaders. New Kensongton: Whitaker House.

Navarro Corona, C. (2016). Consideraciones teóricas sobre el concepto de liderazgo y su aplicación en la investigación educativa. Revista Educación, 40(1), 53-66. DOI: https://doi.org/10.15517/revedu.v40i1.16148

Robinson, V. M., Lloyd, C. A. y Rowe, K. J. (2014). El impacto del liderazgo en los resultados de los estudiantes: un análisis de los efectos diferenciales de los tipos de liderazgo. REICE. Revista Iberoamericana sobre Calidad, Eficacia y Cambio en Educación, 12(4e), 13-40. Recuperado de: http://hdl.handle.net/10486/663297

Ryan, J. y Tipu, S. (2013). Leadership effects on innovation propensity: A two-factor full range leadership model. Journal of Business Research, 66(10), 2116-2129. DOI: https://doi.org/10.1016/j.jbusres.2013.02.038

Salazar Gómez, E. y Tobón, S. (2018). Análisis documental del proceso de formación docente acorde con la sociedad del conocimiento. Revista Espacios, 39(53), 1-17. Recuperado de: http://www.habilidadesparaadolescentes.com/archivos/2018_Analisis_documental_del_proceso_de_formacion_docente_acorde_con_la_sociedad_del_conocimiento.pdf

Sánchez Manchola, I. D. (2008). Los estilos de dirección y liderazgo: propuesta de un modelo de caracterización y análisis. Pensamiento y Gestión, 25, 1-39. Recuperado de: http://www.scielo.org.co/scielo.php?script=sci_ arttext\&pid=S1657-62762008000200002\&lng=en\&tlng=es

Sandoval-Estupiñán, L., Pineda-Báez, C., Bernal-Luque, M.y Quiroga, C. (2020). Los retos del director escolar novel: formación inicial y liderazgo. Revista Complutense de Educación, 31(1), 115-124. DOI: https://doi.org/10.5209/ rced.61919

Santos Estevinha, P. A. (2016). Influência da liderança transformacional e da cultura organizacional na confiança organizacional. Disertación Doctoral, Universidad de La Rioja.

Schmelkes, S. (2011). La calidad depende de todos los que participan en el proceso. Calidad Educativa [portal web]. Obtenido de https://www.calidadeducativa.edusanluis.com.ar/2011/o6/la-calidad-depende-de-todos-los-que.html

Shaaban, S. (2017). The impact of leadership style on middle managers' motivation: A study of the Ministry of Civil Aviation in Egypt. Journal of Business and Management Science, 5(3), 100-107. Recuperado de: http:// www.sciepub.com/reference/219069 
ISSN 0123-1294 | e-ISSN 2027-5358 | Educ.Educ. Vol. 24. No. 2 | Mayo-julio de 2021 | pp. 243-264

Universidad de La Sabana | Facultad de Educación

Sosik, J. J.y Jung, D. (2018). Full Range leadership development: Pathways for people, profit, and planet (2. ed.). Routledge. Recuperado de: https://doi.org/10.4324/9781315167206.

Trógolo, M., Pereyra, A. y Sponton, C. (2013). Impacto de diferentes estilos de liderazgo sobre el Engagement y Burnout: evidencia en una muestra de trabajadores argentinos. Ciencia y Trabajo, 15(48), 152-157. DOI: https://doi.org/10.4067/S0718-24492013000300008

Vega Villa, C. y Zavala V., G. (2004). Adapatación del Cuestionario Multifactorial del Liderazgo (MLQ forma 5 X corta) de B. Bass y B. Avolio al contexto organizacional chileno. [Tesis en Psicología], Universidad de Chile, Santiago.

Zalles, J. H. (2011). Liderazgo: un concepto en evolución. Quito: Konrad Adenauer Stiftung. 